\title{
RAGE is a Potential Cause of Onset and Progression of Nonalcoholic Fatty Liver Disease
}

\author{
Kamyar Asadipooya $\left(\mathbb{D},{ }^{1}\right.$ Kamran B. Lankarani $\left(\mathbb{D},{ }^{2}\right.$ Rishi Raj $\mathbb{D}^{3}{ }^{3}$ \\ and Mohammadreza Kalantarhormozi iD $^{4}$ \\ ${ }^{1}$ Division of Endocrinology and Molecular Medicine, Department of Medicine, University of Kentucky, Lexington, KY, USA \\ ${ }^{2}$ Health Policy Research Center, Institute of Health, Shiraz University of Medical Sciences, Shiraz, Iran \\ ${ }^{3}$ Division of Endocrinology and Molecular Medicine, Department of Medicine, University of Kentucky, Lexington, KY, USA \\ ${ }^{4}$ Endocrinology and Internal Medicine, The Persian Gulf Tropical Medicine Research Center, \\ Bushehr University of Medical Sciences, Bushehr, Iran \\ Correspondence should be addressed to Kamyar Asadipooya; kas224@uky.edu
}

Received 30 January 2019; Revised 5 August 2019; Accepted 26 August 2019; Published 18 September 2019

Academic Editor: Dario Acuña-Castroviejo

Copyright (c) 2019 Kamyar Asadipooya et al. This is an open access article distributed under the Creative Commons Attribution License, which permits unrestricted use, distribution, and reproduction in any medium, provided the original work is properly cited.

\begin{abstract}
Objective. Fatty liver is a rising global health concern, significantly increasing the burden of health care cost. Nonalcoholic fatty liver disease (NAFLD) has a correlation with metabolic syndrome and its complications. Method. We reviewed the literature regarding the mechanisms of developing NAFLD through AGE-RAGE signaling. Results. NAFLD, metabolic syndrome, and production of advanced glycation end-products (AGEs) share many common risk factors and appear to be connected. AGE induces production of the receptor for AGE (RAGE). AGE-RAGE interaction contributes to fat accumulation in the liver leading to inflammation, fibrosis, insulin resistance, and other complications of the fatty liver disease. The immune system, especially macrophages, has an important defense mechanism against RAGE pathway activities. Conclusion. Soluble form of RAGE (sRAGE) has the capability to reduce inflammation by blocking the interaction of AGE with RAGE. However, sRAGE has some limitations, and the best method of usage is probably autotransplantation of transfected stem cells or monocytes, as a precursor of macrophages and Kupffer cells, with a virus that carries sRAGE to alleviate the harmful effects of AGE-RAGE signaling in the settings of fatty liver disease.
\end{abstract}

\section{Introduction}

Nonalcoholic fatty liver disease (NAFLD) refers to the presence of hepatic steatosis in absence of substantial alcohol consumption or any other underlying causes of liver disease $[1,2]$. It is a global health burden and is not only the most common cause of chronic liver disease in the United States but also affects middle- and low-income countries, affecting up to one-third of the adult population [3-5].

NAFLD has a wide clinical and pathological spectrum and ranges from simple fat deposition, nonalcoholic steatohepatitis (NASH), hepatocyte necrosis, and inflammation with or without fibrosis, to the end-stage liver disease that may need liver transplantation $[1,2]$. Although the pathogenesis of
NAFLD is not completely elucidated, hepatic steatosis seems to be an inciting event subsequently leading to inflammation, cell injury, and distortion of the cellular structure through micro- and macrovascular mechanisms $[2,6]$.

Risk factors for NAFLD are almost the same as risk factors for metabolic syndrome and include central obesity, insulin resistance, dysglycemia, dyslipidemia, and hypertension [7]. Dietary factors like increased intake of refined sugars, fructose, saturated fat, and cholesterol as well as decreased intake of polyunsaturated fatty acids especially omega 3 as opposed to omega 6 has been related to NAFLD progression $[7,8]$. Because of its etiopathogenic similarities with metabolic syndrome, NAFLD is also considered as the hepatic component of metabolic syndrome [9]. 
The majority of patients with NAFLD are asymptomatic but can present with nonspecific symptoms such as fatigue, early satiety, and malaise; however, it lacks clear causality [10]. Hepatomegaly can be seen in the advanced stage, and eventually, symptoms and signs of chronic liver disease and portal hypertension ensue $[11,12]$.

NAFLD is diagnosed by evidence of fat accumulation in the liver by either histologic or imaging findings [5]. The histologic threshold is fat deposition in at least $5 \%$ of hepatocytes [13]. Although transabdominal sonography is the most commonly used testing modality with a sensitivity and specificity of $85 \%$ and $95 \%$, respectively, it requires a higher fat accumulation (Up to 20-30\%) for diagnosis and has high inter- and intraobserver variation [14]. The most accurate imaging for evaluation of fat deposition is T1-weighted dualecho magnetic resonance (MR) image and proton magnetic resonance spectroscopy $[15,16]$. The gold standard for diagnosis of inflammation and fibrosis is liver biopsy [14, 17]. The fibrosis (FIB)-4 score and NAFLD fibrosis score which are based on age, serum liver enzymes, serum albumin content, platelet count, and anthropometric measures and presence of diabetes mellitus have shown promise in detecting patients with fibrosis among those suffering from simple steatosis $[15,18]$.

Patients with NAFLD may progress to NASH, cirrhosis, and decompensation in long term $[15,19]$. It is also considered a multisystem disease affecting not only the liver but also the heart and kidney among others [20-22]. Although the progression seems to be secondary to environmental factors and exposure to advanced glycation end-products (AGEs), its possibility, as well as the rate of progression, has remained a matter of debate $[2,6]$.

In this review, a brief discussion regarding the pathophysiology of NAFLD and the role of AGEs in the development and progression of NAFLD into NASH, cirrhosis, and hepatocellular carcinoma has been collected. Finally, we will debate the current opportunities to prevent and/or treat NAFLD and AGE-related liver injury and propose a potential therapeutic approach.

\section{The Process of AGE, RAGE, and sRAGE Production}

Diet plays an important role in the development of NAFLD. Typical dietary pattern of NAFLD patients consists of increased consumption of saturated fatty acid-, cholesterol-, and fructose-rich products. Polyunsaturated fatty acids (PUFAs) and antioxidants have protective roles against NAFLD development [23]. On the contrary, increased intake of fructose and fat-processed foods containing saturated fatty acids are the major sources of endogenous advanced glycation end-products (AGEs). [23, 24]. Based on the correlation between high fat/carbohydrate diets with NAFLD and AGEs production and the protective roles of antioxidants and diets containing PUFA, AGE production may play a crucial role in the onset and progression of NAFLD. Furthermore, aging, type 2 diabetes mellitus, obesity, and metabolic syndrome are reported as risk factors of developing NAFLD which have association with increased AGE production $[23,25,26]$.

Three different pathways participate in endogenous generation of AGEs including nonenzymatic glycation, the polyol pathway, and lipid peroxidation. The process of AGE accumulation usually takes place in the setting of hyperglycemia; however, other conditions such as aging, inflammation, renal failure, oxidative stress, consumption of high fat diet or processed food, excessive alcohol consumption, and cigarette smoking can increase endogenous or exogenous production of AGEs. The initial reaction starts with a nonenzymatic reaction between the reactive carbonyl group of reducing sugar (such as glucose, fructose, glyceraldehyde or carbonyl compounds, and acetaldehyde) and a free amino group on proteins. The reaction is reversible, produces a Schiff's base, and then undergoes spontaneous rearrangement resulting in a partially reversible Amadori product. The nonenzymatic glycation (Maillard) reaction can also happen between reducing sugar and the other macromolecules (lipids and nucleic acids); however, in human subjects with diabetes, the Maillard reaction mainly occurs between sugar, lysine, and arginine amino acid residues. Initially produced AGEs will undergo further modification, which leads to the production of heterogeneous chemical structures that can be found in different tissues and even plasma. AGE accumulation in the tissues affects proteolysis leading to increase in oxidized and damaged proteins, induction of inflammation, increase in reactive oxygen species production, and finally affecting cellular metabolic activities, which may further perpetuate AGE production (Figure 1) [23, 25].

AGEs not only have direct prooxidant and proinflammatory effects but also interact with variable cell surface receptors including receptor for AGEs (RAGE), scavenger receptors including class $A$, class $B$ (CD36), class $E$ (LOX-1), and class $\mathrm{H}$ (FEELs), and AGE receptor complexes (AGE-R). AGEs also induce production of RAGE, leading to initiation of different intrinsic signaling pathways consisting of PKC, PI3K/Akt, Src/RhoA, JAK/STAT, MAPK/ERK, and NADPH oxidase. Activation of these intrinsic signal pathways results in higher levels of NF- $\kappa \mathrm{B}$, Egr-1, and/or other transcription factors which upregulate inflammation, oxidative injury affecting cellular motility, adhesion, and metabolism, ultimately causing tissue injury [24, 25, 27]. In addition, the AGE/RAGE signaling pathway can also activate apoptosis through caspase family (Figure 2) [28, 29].

RAGE is a structurally like immunoglobulin. It has extracellular portion containing V-C1-C2 domains, transmembrane domain, and intracellular domain. The $\mathrm{V}$ domain interacts with RAGE ligands. The cytoplasmic domain of RAGE interacts with diaphanous-1, which is required to activate the postreceptor signaling pathways [30]. DIAPH1 is a type of formin which is principally involved in cytoskeleton, interacts with microtubules, and plays a crucial role in cell organelles kinetics [31]. The interaction between DIAPH1 (mDia1) with RAGE stabilizes RAGE on cell membranes (Figure 3).

The RAGE can be expressed in different tissues and cell types including immune system cells monocyte/ 


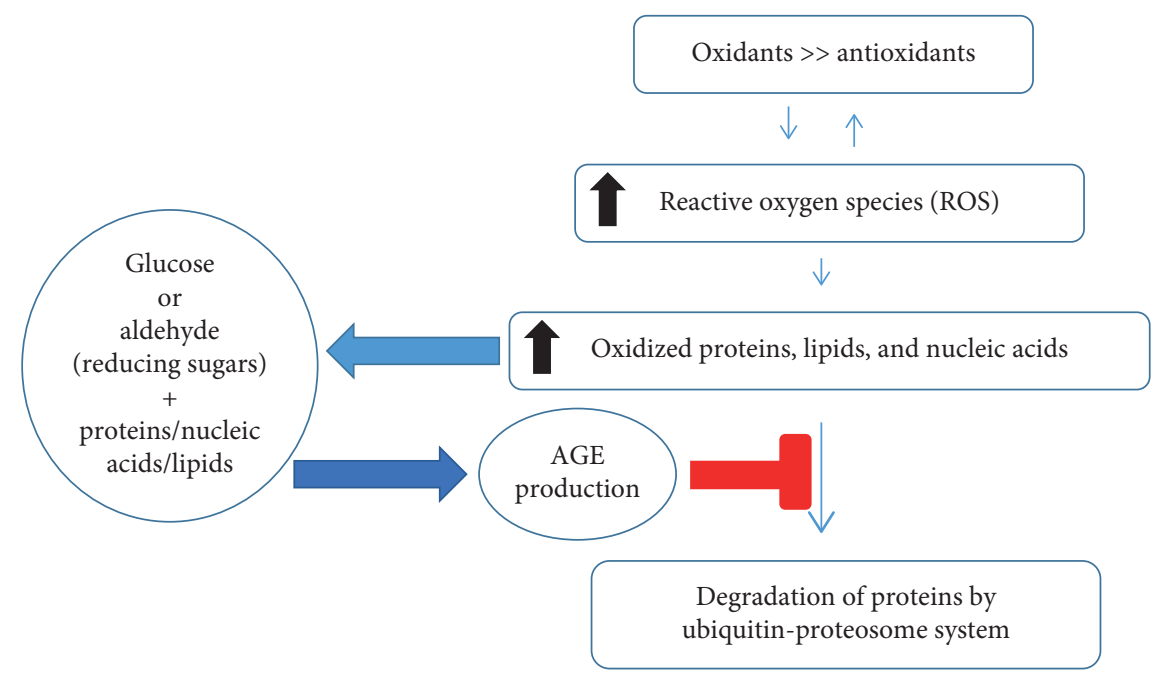

Figure 1: The cycle of AGE production [23].

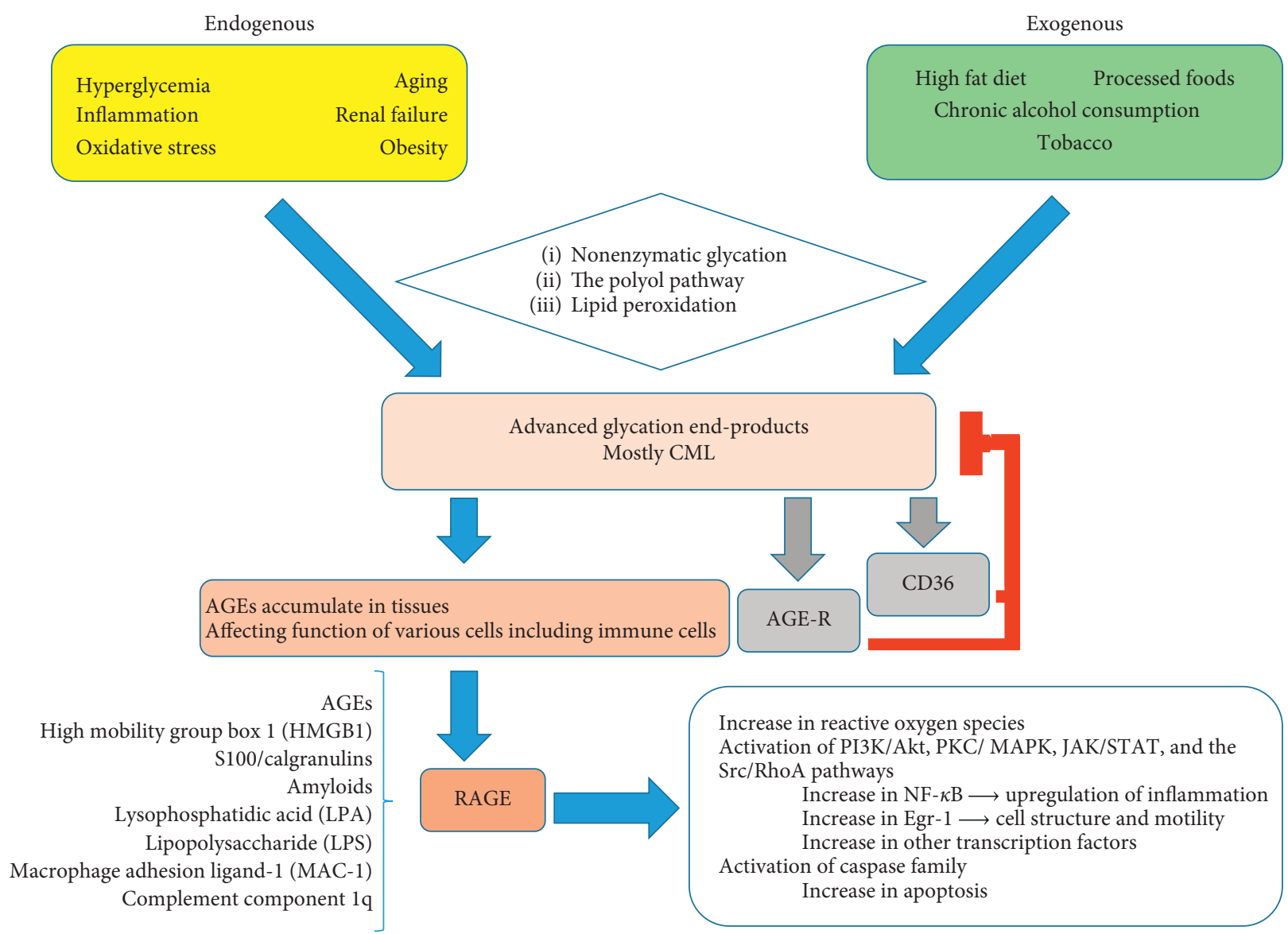

Figure 2: The process of AGE formation, RAGE-ligands interaction, and related consequences. CML, pentosidine, argpyrimidine, imidazolone, GOLD, MOLD, DOLD, carboxyethyl-lysine, fructose-lysine, and methylglyoxal-derived hydroimidazolones are commonly produced AGEs secondary to the reaction between carbohydrate and proteins.

macrophage, lymphocyte, and neutrophil, endothelial cells, smooth muscle cells, and hepatocytes where it interacts with variable ligands $[1,23,25]$. The RAGE ligands include AGEs, HMGB1 (high mobility group box 1), LPS (lipopolysaccharides), S100/calgranulins, amyloid- $\beta$ peptide $(\mathrm{A} \beta)$, and other forms of amyloid, lysophosphatidic acid (LPA), macrophage adhesion ligand-1 (MAC-1), and complement component 1q (C1q) (Figures 2 and 3) [23, 24, 27].

AGEs mediated pathways can be inhibited through AGE-Rs or CD36. While AGE-R detoxifies AGEs, CD36 mediates 


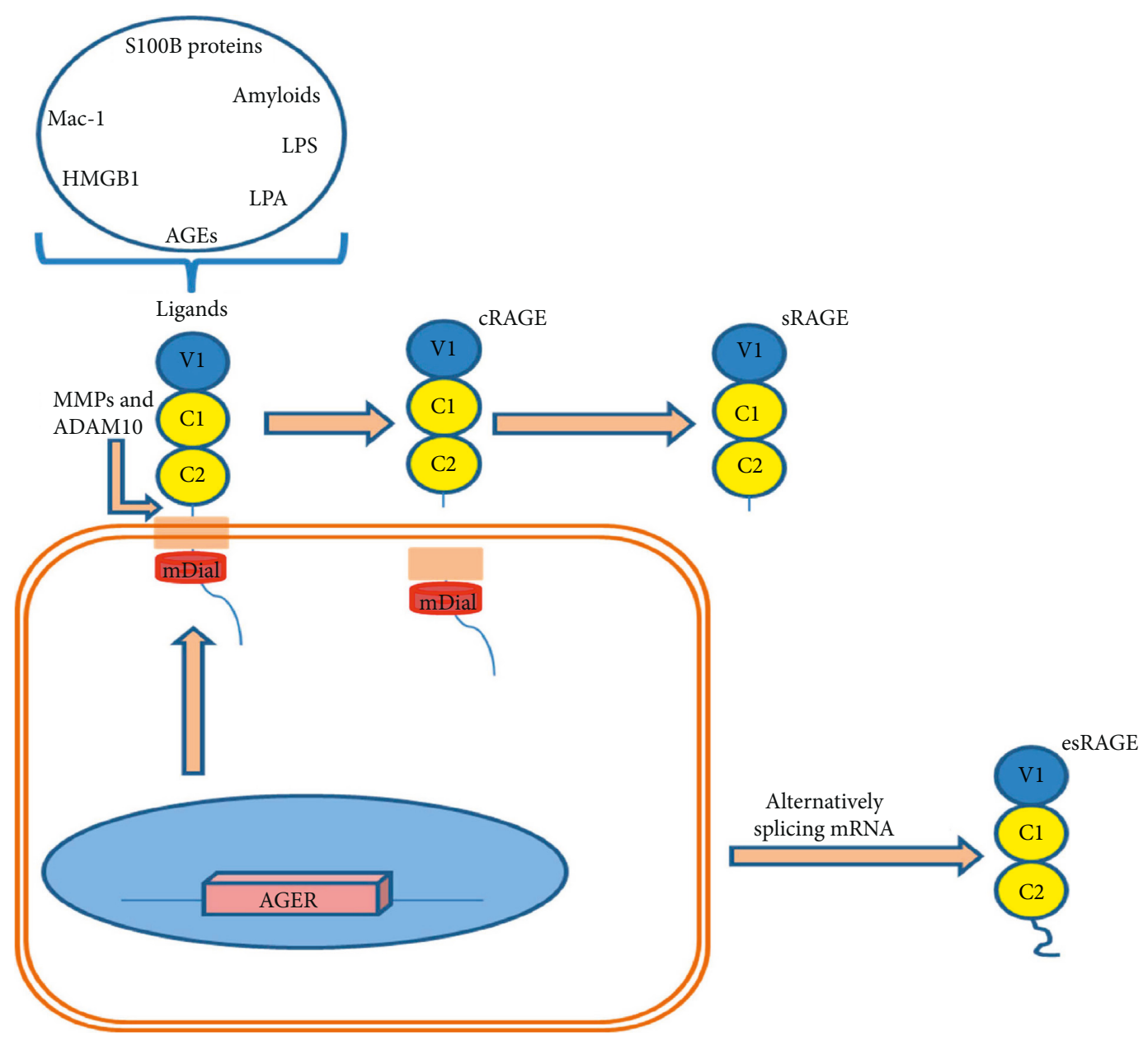

FIGURE 3: RAGE structure and the process of sRAGE formation.

endocytosis of AGEs, leading to intracellular degradation through lysosome and is eventually cleared by the kidneys [23, 24].

RAGE signaling pathway can be potentially inhibited by different mechanisms. First, enzymatic cleavage of RAGE (cRAGE) by ADAM10 (a disintegrin and metalloproteinase domain-containing protein 10) and matrix metalloproteinase (MMPs) disrupts the external domain of the RAGE and leads to the production of soluble RAGE (sRAGE) which is a natural defense tool against AGEs. sRAGE can attach to AGEs and block AGE-RAGE interaction. sRAGE contains the extracellular portion (V-C1-C2 domains) of RAGE molecules and can be measured by the ELIZA method [25, 32]. AGEs can potentially increase production and activity of matrix metalloproteinase activity which technically reduces RAGE signaling activities and increases sRAGE production [33, 34]. Second, endogenous secretory RAGE (esRAGE) which is produced by alternatively spliced pre-mRNA of AGER gene is another form of soluble RAGE that can block AGE-RAGE interaction (Figure 3) [25, 32].

\section{AGE-RAGE Interaction and Development of Fatty Liver}

NAFLD is associated with obesity and diabetes. Liver plays a crucial role in glucose and lipid metabolism. Although the exact pathogenesis of NAFLD has not been clarified, inflammation, oxidant injury, apoptosis, and cell proliferation are the known events in NAFLD which are interconnected with glucose and lipid metabolism. The onset and development hepatic steatosis is linked with insulin resistance [35]. In addition, the complex mixture of metabolic and inflammatory signals causes the course of events from obesity to diabetes, and RAGE signaling pathway might have an important role [36].

The spectrum of NAFLD spans from isolated fat deposition (steatosis) to variable hepatocellular injury due to inflammation with or without fibrosis (NASH). Triglyceride (TG) turnover determines energy homeostasis in human. Excess accumulation of TG in hepatocytes due to increased uptake or de novo synthesis of fatty acids (FA) is an important reason for development of NAFLD [37]. In addition, increased dietary intake of cholesterol can promote fatty liver and inflammation due to mitochondrial dysfunction [35]. Excess lipid storage seems to be an initial step for hepatocellular damage. Overstorage of TG generally is associated with lipotoxicity which leads to cellular organelle dysfunction and injury, ultimately resulting in obesity and related diseases such as fatty liver, metabolic syndrome, and diabetes. Recent literature indicates that lipotoxicity mainly occurs by specific lipids such as free fatty acids (palmitic 
acid), diacylglycerols, cholesterol, ceramides, and lysophosphatidylcholine. Lipotoxicity causes unbalanced energy metabolism that progresses through different cellular signaling cascades including endoplasmic reticulum (ER) stress, mitochondrial dysfunction, induction of oxidative stress, and finally leading to inflammation and cellular apoptosis [37, 38].

The inflammation, cellular proliferation, and increased oxidative stress caused by AGEs lead to progression of NAFLD from simple steatosis to NASH and fibrosis [39]. On the contrary, oxidative stress and inflammation can also cause AGE production [1]. As AGEs induce RAGE signaling, the activated RAGE signaling pathway further induces oxidative stress and inflammation [23,40]. The correlation between RAGE signaling pathway with oxidative stress and inflammation is therefore of a cyclic causation.

RAGE plays an important role in connecting innate and adaptive immune system as well as participates in the onset and maintenance of inflammation [41]. It facilitates cellular adhesion and migration of white blood cells and interacts with some of the released proinflammatory molecules such as HMBG1 and calgranulin/S100 family [42]. Mice experiments have shown that a high fat diet induces the inflammatory response leading to increase in the concentration of RAGE ligands in the liver. In addition, knocking out RAGE attenuates the effects of high fat diet on hepatic glucose production and improves insulin function in the liver [36].

The rate of cell death in the rat liver with diabetes is directly correlated to oxidative stress and expression of HMBG1 which ultimately regulates the cross-talk between apoptosis and autophagy [43]. The rate of progression of liver disease from NAFLD to fibrosis is higher among patients with elevated blood glucose levels, which can be attributed to excess AGEs and RAGE production among these groups of patients $[44,45]$. This signifies the importance of AGEs having a direct involvement in progression of NAFLD through interaction with RAGE [46].

This signifies importance of AGE-RAGE signaling as an important cause of development and progression of NAFLD from simple fat deposition in hepatocytes to development of extensive liver fibrosis.

\section{AGE-RAGE Signaling and Development of Insulin Resistance in NAFLD}

Obesity and insulin resistance are the two most important contributors to the development of metabolic syndrome and NAFLD. Insulin regulates glucose and lipid metabolism. Hepatic insulin resistance is considered as an important sign or cause of NAFLD. Hepatic insulin resistance is defined as impaired suppression of hepatic glucose production through glycogenolysis and/or gluconeogenesis by insulin. It can have several different mechanisms at the molecular level including hyperglycemia with hyperinsulinemia, increased free fatty acids, and their metabolites, oxidative stress, altered profile of adipocytokines and formation of advanced glycation end-products [47].
In addition, high fat diet and endogenous fatty acid synthesis mediated by fatty acid synthase (FAS) are known to be involved in macrophage-induced pathological insulin resistance [48].

High fat diet and hyperglycemia increase formation and accumulation of AGEs. Increased AGEs and its interaction with its cellular receptor RAGE activate cascade of downstream cellular signaling and ultimately causing oxidative stress and chronic inflammation [49]. High AGE levels can also aggravate liver injury by not only the upregulation of inflammation, oxidative stress, and cytokines synthesis but also the activation of hepatic stellate cells that are involved in fat storage and fibrosis [50]. AGEs also disrupt mitochondrial energy metabolism and therefore inhibit cellular proliferation [43]. Glyoxal, which has been connected to oxidative stress and leads to AGEs formation, can cause liver mitochondrial dysfunction in a dose-dependent manner [51]. It also interferes with lipid metabolism and functions of antioxidants [52]. It seems that AGEs have a crucial role in development of hepatic insulin resistance [47, 53, 54]. Furthermore, chronic hyperglycemia and hyperinsulinemia also lead to downregulation of soluble form of RAGE (sRAGE) which again enhances the AGE-RAGE cascade leading to further insulin resistance [55].

Not only AGE-RAGE pathway leads to insulin resistance, but de novo lipid accumulation in hepatocytes also contribute to and can accentuate insulin resistance. Lipid accumulation in hepatocytes impairs intracellular signal transmission upon activation of insulin receptors leading to insulin resistance $[47,56]$. The molecular events comprise progressive mitochondrial dysfunction and autophagy, increase in toxic lipids derivative intermediates (diacylglycerol -DAG- and ceramide), and decrease in total phosphatidylcholine, causing immune-mediated hepatocellular damage, ultimately resulting in hepatic insulin resistance $[37,56]$. Hepatic insulin resistance also drives glucose into lipogenesis instead of glycogen synthesis leading to further hepatocyte lipid accumulation [57].

Despite strong association between AGE-RAGE signaling, insulin resistance, and NAFLD, the process of evolution from NAFLD to NASH, liver cirrhosis, and/or hepatocellular carcinoma is not only influenced by obesity and insulin resistance but also due to integrated interaction of genes, environmental factors, gut microbiome, and immune system [50]. However, presuming the correlation of the same factors contributing to hepatic insulin resistance, AGEs production, and activation of RAGE signaling, we may consider a crucial role for AGE-RAGE signaling in the development of hepatic insulin resistance due to NAFLD.

\section{AGE-RAGE Signaling and Immune Cells, Macrophages}

RAGE expression on immune cells, including macrophages, has been shown. High fat diet increases expression of RAGE ligand in the liver and adipose tissue [36]. The level of AGER mRNA is significantly higher in diabetic versus nondiabetic monocytes from the peripheral blood of human subjects [58]. AGEs have negative effects on the polarization and 
anti-inflammatory roles of M2 macrophages while augmenting the proinflammatory response of M1 macrophages [59].

Furthermore, sRAGE, a RAGE ligand decoy, has a promising result on reducing inflammation and prolongation of rat survival after acute liver failure and could improve hepatocytes survival after ischemic reperfusion injury $[40,60]$. However, absence of RAGE on macrophages or applying sRAGE can diminish macrophage phagocytosis and clearance of apoptotic cells which is important for tissue homeostasis and modulation of inflammation [61]. On the contrary, transfection of bone marrow mesenchymal stem cells or umbilical cord-derived mesenchymal stem cells by sRAGE has more suppressive effects on inflammation and improves cell survival $[60,62]$. This probably means that the transfected mesenchymal cells provide more accessible sRAGE to the area of AGE-RAGE signaling without disabling immune cells function. In other words, it seems that the cross-talk between AGE-RAGE signaling and inflammation/insulin resistance is complex, and probably initial activation of macrophages has beneficial effects, but further activation can lead to more inflammation and insulin resistance.

\section{6. sRAGE and Its Correlation with NAFLD}

sRAGE competes with RAGE ligands in binding to RAGE on cell membranes but does not initiate signal transduction, and thereby, it has a protective role in NAFLD. The serum levels of sRAGE in NAFLD patients are lower than the general population, and its level correlates with severity of hepatic steatosis, with higher levels of sRAGE in patients with mild disease compared to patients with moderate-tosevere hepatic steatosis [63]. Many articles have reported correlation of sRAGE and/or esRAGE with various diseases or conditions; however, the data are inconsistent. There appears to be a bidirectional correlation, which further depends on the state and/or extent of the disease process. For instance, sRAGE has a negative correlation with body mass index (BMI) and rises with antidiabetic medications (thiazolidinediones) and after bariatric surgery [64-66]. Lower levels of sRAGE are reported in atherosclerosis, hypercholesterolemia, and hypertension, whereas higher levels are found in patients with diabetes [27]. Therefore, sRAGE cannot be a reliable biomarker to assess the RAGE signaling activities as it can be elevated in either excess RAGE production or disruption. However, according to the fact that AGEs induce ADAM10 secretion, AGEs/sRAGE ratio might be helpful in the diagnosis of NAFLD patients (Table 1) $[67,78,79]$.

\section{Current Treatment, Targeting RAGE, and Future Direction}

Despite significant improvement in the understanding and knowledge of etiology and pathophysiology of NAFLD, there is still no treatment modality which has been proven to be successful in the treatment of NAFLD. Life-style modifications, such as exercise and weight loss, remain the cornerstone of treatment for patients with NAFLD and NASH. Pharmacologic therapy is generally recommended for biopsy-proven NASH and high-risk situations such as liver cirrhosis and/or treating complications, such as diabetes, hypertension, or hyperlipidemia. Biguanide (metformin), peroxisome proliferator-activated receptor gamma (PPAR- $\gamma$ ) agonists (pioglitazone), long-acting glucagon-like peptide- (GLP-) 1 receptor agonist (liraglutide), vitamin E, lipid-lowering agents (such as statins, ezetimibe, and fibrates), antioxidants, pentoxifylline, angiotensin receptor blockers (losartan), ursodeoxycholic acid (UDCA), symbiotic or probiotics, obeticholic acid (Farnesoid X receptor agonist), and peroxisome proliferator-activated receptor alpha (PPAR- $\alpha$ ) agonist (Elafibranor) are currently available medical therapy for treatment of NAFLD. Reduction in inflammation, fibrosis, lipid production, and hepatocellular injury by these medications leads to improvement in fatty liver disease [80]. However, these medications also play a role in reducing AGE production and/or RAGE expression, emphasizing the importance of AGE-RAGE signaling in initiation and progression of NAFLD [81-85].

As increased production of AGEs, inducing RAGE production and resulting in activation of AGE-RAGE signaling with its downstream effects at intra- and extracellular levels appear to be central in the development and progression of NAFLD, targeting AGE-RAGE signaling with AGE inhibitor (aminoguanidine), sRAGE (the ligand decoy), anti-RAGE antibody, small molecule RAGE antagonists aptamers, inhibitors of cytoplasmic domain of RAGE (ctRAGE), nanocarriers for RAGE siRNA therapy, and/or inhibitors of the HMGB1-RAGE interaction appear to be extremely promising and can potentially reduce inflammation, improve cell survival, and prevent development and/or progression of NAFLD [27, 86-89]. AGEs are structurally modified molecules, which induce inflammation after its interaction with RAGE, and therefore blocking AGEs, by means of sRAGE, and can prevent the AGE-RAGE interaction [23]. However, sRAGE does not cross the bloodbrain barrier and has no significant effects on physical activity, appetite, or energy expenditure. Intraperitoneal injection of sRAGE results in significant differences in body mass between sRAGE versus vehicle-treated high fat-fed mice. sRAGE could improve insulin sensitivity and reduce inflammation. Nevertheless, sRAGE has had some limitations in blocking AGEs. It increases the levels of HMGB1 (RAGE ligand) mRNA in the liver tissue of high fat-fed mice [36]. Heterogeneity of AGEs, accessibility of sRAGE, and possible loss of beneficial effects of AGE-RAGE signaling by using sRAGE may further lessen the therapeutic effects of recombinant sRAGE [79].

Macrophages have a crucial role in initiation and maintenance of inflammation. In addition, the process of liver lipotoxicity seems to be caused by accumulation triglyceride-derived material, followed by inflammation and cellular dysfunction, which needs activity of macrophages and other immune cells [90]. Free fatty acid can increase liver macrophage accumulation which technically reiterates the process of fat accumulation as well [91]. Furthermore, the process of lobular inflammation in the liver, due to 
TABLE 1: Clinical studies about AGE/RAGE and NAFLD/NASH in reverse order of the publication year.

Author/Journal and year
Palma-Duran et al., Metabolism,
2018 Jun. [67]
Mehta et al., PLoS One, 2018 Jun.
[68]
Zelber-Sagi et al., Dig Liver, Dis,
2017 May [63]

Kan et al., J Clin Lab Anal, 2015 Nov. [69]

Santilli et al., Vascular Pharmacology, 2015 Sep. [70]

Su et al., Medicine (Baltimore), 2015 Aug. [71]

D’Adamo et al., Free Radic Res, 2013 Mar. [72]

Gaens et al., Journal of Hepatology, 2012 Mar. [73]

D'Adamo et al., Antioxidants and Redox signaling, 2011 Mar. [74]

Kimura et al., J Gastroenterol, 2010 Jul. [75]

Yilmaz et al., Clin Biochem, 2009 Jun. [76]

Hyogo et al., J Gastroenterol Hepatol, 2007 Jul. [77]

Participants

50 normoglycemic NAFLD and

58 healthy adults (age, sex, and BMI matched)

340 obese patients with metabolic syndrome

55 patients with fatty liver diagnosed by ultrasound (age 26-64)

\section{NBNC-HCC $56 \mathrm{NASH}$}

27 control subjects

110 patients with FCHL, MS, and both FCHL plus MS

300 cancer-free cases

265 HCC patients

Obese prepubertal children with NAFLD

(i) 24 children received vitamin $\mathrm{E}$ supplementation

(ii) 21 age- and sex-matched received life-style intervention

74 obese individuals

140 prepubertal obese children

71 boys and 69 girls aged
between 6 and 10 years

43 patients with dyslipidemia and biopsy-proven NASH $40 \mathrm{NASH}$

8 borderline NASH

9 simple fatty liver

14 healthy controls $66 \mathrm{NASH}$

10 simple steatosis

30 controls
Elevated AGEs/sRAGE ratio was associated with NAFLD

Certain polymorphisms of RAGE gene are indicators of NASH in obese people

sRAGE levels have negative correlation with liver damage

AGE could discriminate NBNCHCC from NASH

NAFLD patients had significantly lower plasma esRAGE irrespective of underlying metabolic abnormality (FCHL or MS)

SNP rs1800625 of RAGE gene increases risk of HCC but has an inverse association with HCC progression

Vitamin E supplementation increased the levels of esRAGE after 6-month treatment

Vitamin E supplementation decreased oxidative stress and improved cardiometabolic alterations

CML and CML upregulated RAGE-dependent inflammatory response associated with NAFLD

esRAGE and sRAGE levels were significantly lower in obese prepubertal children affected by liver steatosis compared with the group without liver steatosis

Atorvastatin reduces the serum levels of AGEs

Patients with NASH (definite and borderline) had lower plasma levels of sRAGE

The serum glyceraldehydederived AGE level may be a useful biomarker for NASH
AGEs (CML) higher, sRAGE

levels lower, and AGEs/sRAGE ratios were higher in the NAFLD Retrospective study

Only four polymorphisms were tested

Serum sRAGE levels have negative association with fatty liver and positive correlation with fiber and vegetables consumption

NBNC-HCC patients had higher

serum levels of AGE, compared

to the NASH and control subjects

Cross-sectional study

Lower levels of IL-10 and adiponectin and higher CD40 ligand, endogenous thrombin potential, and oxidized LDL in NAFLD patients

5 common polymorphisms of RAGE gene were tested

Vitamin E could potentially be a treatment in obese children with NAFLD

In vitro model of steatosis used CML accumulation associated with increased gene expression of proinflammatory cytokines

Definition of liver steatosis by ultrasound instead of the goldstandard invasive liver biopsy

AGEs are useful biomarkers for the attenuation of NASH

Constant increase in glyceraldehyde-derived AGEs could potentially contribute to the pathogenesis of NASH inflammatory cell infiltration and secretion of cytokines, can lead to necrosis and progression of NAFLD to NASH. However, macrophages are the resident inflammatory cells, which are involved in initiation and progression of inflammation [92].

AGE-RAGE signaling pathway plays an important role in recruiting macrophages in inflammation and inducing oxidative stress, but AGEs can increase lipid accumulation in macrophages, which disables macrophages [25, 79, 93]. In addition, AGE-RAGE signaling not only participates in insulin resistance but also seems to have some defensive role, according to the fact that knocking out RAGE leads to lower lean mass in high fat diet-fed mice [36]. Furthermore, sRAGE infusion can alleviate inflammation and macrophage phagocytosis $[60,61]$. It seems AGE-RAGE signaling pathway is important for initial activation and phagocytic 
ability of macrophages, but further activity of the pathway may cause unregulated immune cells activity, such as macrophages, which ended up having more inflammation and tissue damage. As a result, transfecting macrophages, as a resident cell, by sRAGE may not prevent initial activation, but then after macrophages got activated, sRAGE can be secreted and probably prevent unregulated stimulation of immune cells.

Based on the capability of sRAGE in decreasing inflammation [60], the important role of macrophages, as a resident inflammatory cell, in the initiation/modulation of inflammation, reduction of macrophage phagocytosis without RAGE [61], and better suppressive effects of secreted sRAGE on inflammation and cell survival after transfection of bone marrow mesenchymal stem cells [60] or umbilical cord-derived mesenchymal stem cells [62], we may propose that watching diet and autotransplantation of transfected stem cells, or probably monocytes as a precursor of liver macrophages, by mutated lentivirus that carries sRAGE would be a reasonable approach to reduce insulin resistance and fat deposition in the liver [36, 60,61]. Theoretically, transfected macrophages, with sRAGE, can be activated initially by being exposed to AGEs. Subsequently, transfected macrophage secretes sRAGE, blocks AGE-RAGE interaction, inhibits further activation, and reduces inflammation, fat deposition, insulin resistance, and hepatocytes injury. However, sRAGE has no effect on energy expenditure or appetite [36], may increase the levels of HMGB1 mRNA in the liver tissue of high fat-fed mice, and HMGB1 as a RAGE ligand, and has some roles in the pathogenesis of liver fibrosis through the RAGE signaling pathway $[36,67]$. On the contrary, the cross-talk between oxidative stress and AGEs formation, which leads to increase in the level of AGEs, may limit the effectivity of the sRAGE. In addition, AGEs are generally complex and heterogenic in nature, which may diminish the effectivity of sRAGE in blocking AGE-RAGE interaction [23]. However, the association of certain RAGE gene polymorphisms with NASH and diabetes may indicate the dominant role of certain AGEs in the pathophysiology of NASH and diabetes, which brings up a potential hope to make a sRAGE against the dominant AGE to be able to block AGE-RAGE interaction [68, 94-96].

\section{Conclusion}

Autotransplantation of transfected stem cells or monocytes as a precursor of macrophages or Kupffer cell, with lentivirus that carries sRAGE, in addition to low carbohydrate/fat diet may give us promising results in treating NAFLD and its complications. However, we should consider limitations of sRAGE, such as accessibility, AGEs heterogeneity, and crosstalk with other RAGE ligands or oxidative stress, as a potential therapeutic approach.

\section{Abbreviations}

ADAM10: A disintegrin and metalloproteinase domaincontaining protein 10

AGE: $\quad$ Advanced glycation end-products
AGE-R: $\quad$ AGE receptor complexes

A $\beta$ : $\quad$ Amyloid- $\beta$ peptide

BMI: $\quad$ Body mass index

CAP: $\quad$ Controlled attenuation parameter

C1q: $\quad$ Complement component 1q

CML: $\quad N \varepsilon$-(carboxymethyl)lysine

cRAGE: Cleaved RAGE

ER: $\quad$ Endoplasmic reticulum

esRAGE: Endogenous soluble RAGE

FAS: $\quad$ Fatty acid synthase

FCHL: $\quad$ Familial combined hyperlipidemia

HCC: Hepatocellular carcinoma

HMGB1: High mobility group box 1

LPA: $\quad$ Lysophosphatidic acid

LPS: $\quad$ Lipopolysaccharides

MAC-1: $\quad$ Macrophage adhesion ligand-1

MMP: $\quad$ Matrix metalloproteinase

MS: $\quad$ Metabolic syndrome

MUFA: $\quad$ Monounsaturated fatty acids

NAFLD: Nonalcoholic fatty liver disease

NASH: Nonalcoholic steatohepatitis

NBNC- Non-B or non-C hepatocellular carcinoma

HCC:

RAGE: Receptor for advanced glycation end-products

SNP: $\quad$ Single-nucleotide polymorphisms

sRAGE: Soluble RAGE

TG: $\quad$ Triglyceride.

\section{Conflicts of Interest}

All authors declare that they have no conflicts of interest.

\section{Authors' Contributions}

Kamyar Asadipooya contributed for reviewing the literature, preparing tables and figures, and writing the manuscript. Kamran Bagheri Lankarani participated in reviewing the literature and writing the manuscript. Rishi Raj helped in preparing the table and editing the manuscript. Mohammadreza Kalantarhormozi helped in editing the manuscript. All authors approved the final version of the manuscript.

\section{References}

[1] M. Takeuchi, J. I. Takino, A. Sakasai-Sakai, T. Takata, and M. Tsutsumi, "Toxic AGE (TAGE) theory for the pathophysiology of the onset/progression of NAFLD and ALD," Nutrients, vol. 9, no. 6, p. 634, 2017.

[2] L. Antonucci, C. Porcu, G. Iannucci, C. Balsano, and B. Barbaro, "Non-alcoholic fatty liver disease and nutritional implications: special focus on copper," Nutrients, vol. 9, no. 10 , p. 1137, 2017.

[3] K. B. Lankarani, F. Ghaffarpasand, M. Mahmoodi et al., "Non alcoholic fatty liver disease in southern Iran: a population based study," Hepatitis Monthly, vol. 13, no. 5, p. e9248, 2013.

[4] I. Moghaddasifar, K. B. Lankarani, M. Moosazadeh et al., "Prevalence of non-alcoholic fatty liver disease and its related factors in Iran," International Journal of Organ Transplantation Medicine, vol. 7, pp. 149-160, 2016. 
[5] S. Bellentani, "The epidemiology of non-alcoholic fatty liver disease," Liver International, vol. 37, no. 1, pp. 81-84, 2017.

[6] A. Zhuang, F. Y. Yap, C. Bruce et al., "Increased liver AGEs induce hepatic injury mediated through an OST48 pathway," Scientific Reports, vol. 7, no. 1, p. 12292, 2017.

[7] B. Honarvar, K. Bagheri Lankarani, P. Keshani, and T. Rafiee, "Dietary determinants of non-alcoholic fatty liver disease in lean and non-lean adult patients: a population-based study in shiraz, southern Iran," Hepatitis Monthly, vol. 17, no. 4, Article ID e12295, 2017.

[8] R. Loomba, V. Seguritan, W. Li et al., "Gut microbiome-based metagenomic signature for non-invasive detection of advanced fibrosis in human nonalcoholic fatty liver disease," Cell Metabolism, vol. 25, no. 5, pp. 1054-1062.e1055, 2017.

[9] S. Sookoian and C. J. Pirola, "Systematic review with metaanalysis: risk factors for non-alcoholic fatty liver disease suggest a shared altered metabolic and cardiovascular profile between lean and obese patients," Alimentary Pharmacology and Therapeutics, vol. 46, no. 2, pp. 85-95, 2017.

[10] K. E. Corey, S. Gawrieh, A. S. deLemos et al., "Risk factors for hepatocellular carcinoma in cirrhosis due to nonalcoholic fatty liver disease: a multicenter, case-control study," World Journal of Hepatology, vol. 9, no. 7, pp. 385-390, 2017.

[11] European Association for the Study of the Liver (EASL), European Association for the Study of Diabetes (EASD), and European Association for the Study of Obesity (EASO), "EASL-EASD-EASO clinical practice guidelines for the management of non-alcoholic fatty liver disease," Obesity Facts, vol. 9, no. 2, pp. 65-90, 2016.

[12] L. Vigano, A. Lleo, and A. Aghemo, "Non-alcoholic fatty liver disease, non-alcoholic steatohepatitis, metabolic syndrome and hepatocellular carcinoma-a composite scenario," Hepatobiliary Surgery and Nutrition, vol. 7, pp. 130-133, 2018.

[13] R. Hernaez, M. Lazo, S. Bonekamp et al., "Diagnostic accuracy and reliability of ultrasonography for the detection of fatty liver: a meta-analysis," Hepatology, vol. 54 , no. 3, pp. $1082-$ 1090, 2011.

[14] S. Petta, V. W.-S. Wong, C. Cammà et al., "Improved noninvasive prediction of liver fibrosis by liver stiffness measurement in patients with nonalcoholic fatty liver disease accounting for controlled attenuation parameter values," Hepatology, vol. 65, no. 4, pp. 1145-1155, 2017.

[15] P. Bedossa, "Pathology of non-alcoholic fatty liver disease," Liver International, vol. 37, no. 1, pp. 85-89, 2017.

[16] E. M. Brunt and D. E. Kleiner, "Challenges in the hepatic histopathology in non-alcoholic fatty liver disease," Gut, vol. 66, no. 9, pp. 1539-1540, 2017.

[17] E. Vilar-Gomez and N. Chalasani, "Non-invasive assessment of non-alcoholic fatty liver disease: clinical prediction rules and blood-based biomarkers," Journal of Hepatology, vol. 68, no. 2, pp. 305-315, 2018.

[18] J. B. Schwimmer, C. Behling, J. E. Angeles et al., "Magnetic resonance elastography measured shear stiffness as a biomarker of fibrosis in pediatric nonalcoholic fatty liver disease," Hepatology, vol. 66, no. 5, pp. 1474-1485, 2017.

[19] J. D. Browning, K. S. Kumar, M. H. Saboorian, and D. L. Thiele, "Ethnic differences in the prevalence of cryptogenic cirrhosis," American Journal of Gastroenterology, vol. 99, no. 2, pp. 292-298, 2004.

[20] N. Chalasani, Z. Younossi, J. E. Lavine et al., "The diagnosis and management of nonalcoholic fatty liver disease: practice guidance from the American Association for the Study of Liver Diseases," Hepatology, vol. 67, no. 1, pp. 328-357, 2018.
[21] P. Dongiovanni and L. Valenti, “A nutrigenomic approach to non-alcoholic fatty liver disease," International Journal of Molecular Sciences, vol. 18, no. 7, p. 1534, 2017.

[22] G. Targher and C. D. Byrne, "Non-alcoholic fatty liver disease: an emerging driving force in chronic kidney disease," Nature Reviews Nephrology, vol. 13, no. 5, pp. 297-310, 2017.

[23] C. Ott, K. Jacobs, E. Haucke, A. Navarrete Santos, T. Grune, and A. Simm, "Role of advanced glycation end products in cellular signaling," Redox Biology, vol. 2, pp. 411-429, 2014.

[24] R. López- Díez, A. Shekhtman, R. Ramasamy, and A. M. Schmidt, "Cellular mechanisms and consequences of glycation in atherosclerosis and obesity," Biochimica et Biophysica Acta (BBA)Molecular Basis of Disease, vol. 1862, no. 12, pp. 2244-2252, 2016.

[25] A. M. Schmidt, "2016 ATVB plenary lecture: receptor for advanced glycation endproducts and implications for the pathogenesis an treatment of cardiometabolic disorders: spotlight on the macrophage," Arteriosclerosis, Thrombosis, and Vascular Biology, vol. 37, pp. 613-621, 2017.

[26] E. Tsai and T.-P. Lee, "Diagnosis and evaluation of nonalcoholic fatty liver disease/nonalcoholic steatohepatitis, including noninvasive biomarkers and transient elastography," Clinics in Liver Disease, vol. 22, no. 1, pp. 73-92, 2018.

[27] E. Litwinoff, C. Hurtado Del Pozo, R. Ramasamy, and A. Schmidt, "Emerging targets for therapeutic development in diabetes and its complications: the RAGE signaling pathway," Clinical Pharmacology and Therapeutics, vol. 98, no. 2, pp. 135-144, 2015.

[28] Q. Wu, Z.-M. Zhong, S.-Y. Zhu et al., “Advanced oxidation protein products induce chondrocyte apoptosis via receptor for advanced glycation end products-mediated, redox-dependent intrinsic apoptosis pathway," Apoptosis, vol. 21, no. 1, pp. 36-50, 2016.

[29] J. Liu, J. Mao, Y. Jiang et al., "AGEs induce apoptosis in rat osteoblast cells by activating the caspase- 3 signaling pathway under a high-glucose environment in vitro," Applied Biochemistry and Biotechnology, vol. 178, no. 5, pp. 1015-1027, 2016.

[30] B. I. Hudson, A. Z. Kalea, M. Del Mar Arriero et al., "Interaction of the RAGE cytoplasmic domain with diaphanous1 is required for ligand-stimulated cellular migration through activation of Rac1 and Cdc42," Journal of Biological Chemistry, vol. 283, no. 49, pp. 34457-34468, 2008.

[31] Y. N. Lin and S. Windhorst, "Diaphanous-related formin 1 as a target for tumor therapy," Biochemical Society Transactions, vol. 44, pp. 1289-1293, 2016.

[32] A. Kumar Pasupulati, P. S. Chitra, and G. B. Reddy, “Advanced glycation end products mediated cellular and molecular events in the pathology of diabetic nephropathy," Biomolecular Concepts, vol. 7, pp. 293-309, 2016.

[33] A. I. Serban, L. Stanca, O. I. Geicu, M. C. Munteanu, M. Costache, and A. Dinischiotu, "Extracellular matrix is modulated in advanced glycation end products milieu via a RAGE receptor dependent pathway boosted by transforming growth factor- $\beta 1$," Journal of Diabetes, vol. 7 , no. 1 , pp. 114-124, 2015.

[34] W. Li, W. Ling, X. Teng, C. Quan, S. Cai, and S. Hu, "Effect of advanced glycation end products, extracellular matrix metalloproteinase inducer and matrix metalloproteinases on type-I collagen metabolism," Biomedical Reports, vol. 4, no. 6, pp. 691-693, 2016.

[35] I. Reccia, J. Kumar, C. Akladios et al., "Non-alcoholic fatty liver disease: a sign of systemic disease," Metabolism, vol. 72, pp. 94-108, 2017. 
[36] F. Song, C. Hurtado del Pozo, R. Rosario et al., "RAGE regulates the metabolic and inflammatory response to high-fat feeding in mice," Diabetes, vol. 63, no. 6, pp. 1948-1965, 2014.

[37] A. Engin, "Non-alcoholic fatty liver disease," Obesity and Lipotoxicity, vol. 960, pp. 443-467, 2017.

[38] F. Marra and G. Svegliati-Baroni, "Lipotoxicity and the gutliver axis in NASH pathogenesis," Journal of Hepatology, vol. 68, no. 2, pp. 280-295, 2018.

[39] R. Patel, S. S. Baker, W. Liu et al., "Effect of dietary advanced glycation end products on mouse liver," PLoS One, vol. 7, no. 4, Article ID e35143, 2012.

[40] S. Zeng, N. Feirt, M. Goldstein et al., "Blockade of receptor for advanced glycation end product (RAGE) attenuates ischemia and reperfusion injury to the liver in mice," Hepatology, vol. 39, no. 2, pp. 422-432, 2004.

[41] T. Kawai and S. Akira, "The role of pattern-recognition receptors in innate immunity: update on Toll-like receptors," Nature Immunology, vol. 11, no. 5, pp. 373-384, 2010.

[42] K. Kierdorf and G. Fritz, "RAGE regulation and signaling in inflammation and beyond," Journal of Leukocyte Biology, vol. 94, no. 1, pp. 55-68, 2013.

[43] A. Petrovic, D. Bogojevic, A. Korac et al., "Oxidative stressdependent contribution of HMGB1 to the interplay between apoptosis and autophagy in diabetic rat liver," Journal of Physiology and Biochemistry, vol. 73, pp. 511-521, 2017.

[44] L. A. Adams, S. Sanderson, K. D. Lindor, and P. Angulo, "The histological course of nonalcoholic fatty liver disease: a longitudinal study of 103 patients with sequential liver biopsies," Journal of Hepatology, vol. 42, no. 1, pp. 132-138, 2005.

[45] V. Ratziu, M. Munteanu, F. Charlotte, L. Bonyhay, and T. Poynard, "Fibrogenic impact of high serum glucose in chro nic hepatitis C," Journal of Hepatology, vol. 39, no. 6, pp. 1049-1055, 2003.

[46] C. Leung, C. B. Herath, Z. Jia et al., "Dietary advanced glycation end-products aggravate non-alcoholic fatty liver disease," World Journal of Gastroenterology, vol. 22, no. 35, pp. 8026-8040, 2016.

[47] I. A. Leclercq, A. Da Silva Morais, B. Schroyen, N. Van Hul, and A. Geerts, "Insulin resistance in hepatocytes and sinusoidal liver cells: mechanisms and consequences," Journal of Hepatology, vol. 47, no. 1, pp. 142-156, 2007.

[48] X. Wei, H. Song, L. Yin et al., "Fatty acid synthesis configures the plasma membrane for inflammation in diabetes," Nature, vol. 539, no. 7628, pp. 294-298, 2016.

[49] H. Unoki and S.-i. Yamagishi, "Advanced glycation end products and insulin resistance," Current Pharmaceutical Design, vol. 14, no. 10, pp. 987-989, 2008.

[50] M. Sharma, S. Mitnala, R. K. Vishnubhotla, R. Mukherjee, D. N. Reddy, and P. N. Rao, "The riddle of nonalcoholic fatty liver disease: progression from nonalcoholic fatty liver to nonalcoholic steatohepatitis," Journal of Clinical and Experimental Hepatology, vol. 5, no. 2, pp. 147-158, 2015.

[51] M. Goudarzi, H. Kalantari, and M. Rezaei, "Glyoxal toxicity in isolated rat liver mitochondria," Human \& Experimental Toxicology, vol. 37, no. 5, pp. 532-539, 2018.

[52] M. Aragno and R. Mastrocola, "Dietary sugars and endogenous formation of advanced glycation endproducts: emerging mechanisms of disease," Nutrients, vol. 9, 2017.

[53] J. J. DiNicolantonio, V. Mehta, N. Onkaramurthy, and J. H. O'Keefe, "Fructose-induced inflammation and increased cortisol: a new mechanism for how sugar induces visceral adiposity," Progress in Cardiovascular Diseases, vol. 61, no. 1, pp. 3-9, 2018.
[54] E. A. Muellenbach, C. J. Diehl, M. K. Teachey et al., "Interactions of the advanced glycation end product inhibitor pyridoxamine and the antioxidant a-lipoic acid on insulin resistance in the obese Zucker rat," Metabolism, vol. 57, no. 10, pp. 1465-1472, 2008.

[55] S. K. Biswas, S. Mohtarin, S. R. Mudi et al., "Relationship of soluble RAGE with insulin resistance and beta cell function during development of type 2 diabetes mellitus," Journal of Diabetes Research, vol. 2015, Article ID 150325, 6 pages, 2015.

[56] R. H. Hassan, O. Bourron, and E. Hajduch, "Defect of insulin signal in peripheral tissues: important role of ceramide," World Journal of Diabetes, vol. 5, no. 3, pp. 244-257, 2014.

[57] V. T. Samuel and G. I. Shulman, "Nonalcoholic fatty liver disease as a nexus of metabolic and hepatic diseases," Cell Metabolism, vol. 27, no. 1, pp. 22-41, 2018.

[58] X.-d. Su, S.-s. Li, Y.-q. Tian, Z.-y. Zhang, G.-z. Zhang, and L.-x. Wang, "Elevated serum levels of advanced glycation end products and their monocyte receptors in patients with type 2 diabetes," Archives of Medical Research, vol. 42, no. 7, pp. 596-601, 2011.

[59] Q. Wang, G. Zhu, X. Cao, J. Dong, F. Song, and Y. Niu, "Blocking AGE-RAGE signaling improved functional disorders of macrophages in diabetic wound," Journal of Diabetes Research, vol. 2017, Article ID 1428537, 10 pages, 2017.

[60] J. Wang, H. Wang, J. Shi, and Y. Ding, "Effects of bone marrow MSCs transfected with sRAGE on the intervention of HMGB1 induced immuno-inflammatory reaction," International Journal of Clinical and Experimental Pathology, vol. 8, no. 10, pp. 12028-12040, 2015.

[61] M. He, H. Kubo, K. Morimoto et al., "Receptor for advanced glycation end products binds to phosphatidylserine and assists in the clearance of apoptotic cells," EMBO Reports, vol. 12, no. 4, pp. 358-364, 2011.

[62] M. Son, S. Oh, H. Park et al., "Protection against RAGEmediated neuronal cell death by sRAGE-secreting human mesenchymal stem cells in 5xFAD transgenic mouse model," Brain, Behavior, and Immunity, vol. 66, pp. 347-358, 2017.

[63] S. Zelber-Sagi, F. Salomone, I. Kolodkin-Gal et al., "Protective role of soluble receptor for advanced glycation end-products in patients with non-alcoholic fatty liver disease," Digestive and Liver Disease, vol. 49, no. 5, pp. 523-529, 2017.

[64] J. M. Brix, F. Hollerl, H. P. Kopp, G. H. Schernthaner, and G. Schernthaner, "The soluble form of the receptor of advanced glycation endproducts increases after bariatric surgery in morbid obesity," International Journal of Obesity, vol. 2012, no. 36, pp. 1412-1417, 2005.

[65] G. D. Norata, K. Garlaschelli, L. Grigore et al., "Circulating soluble receptor for advanced glycation end products is inversely associated with body mass index and waist/hip ratio in the general population," Nutrition, Metabolism and Cardiovascular Diseases, vol. 19, no. 2, pp. 129-134, 2009.

[66] K. C. B. Tan, W. S. Chow, A. W. K. Tso et al., "Thiazolidinedione increases serum soluble receptor for advanced glycation end-products in type 2 diabetes," Diabetologia, vol. 50, no. 9, pp. 1819-1825, 2007.

[67] S. A. Palma-Duran, M. D. Kontogianni, A. Vlassopoulos et al., "Serum levels of advanced glycation end-products (AGEs) and the decoy soluble receptor for AGEs (sRAGE) can identify non-alcoholic fatty liver disease in age-, sex- and BMImatched normo-glycemic adults," Metabolism, vol. 83, pp. 120-127, 2018.

[68] R. Mehta, G. Shaw, P. Masschelin et al., "Polymorphisms in the receptor for advanced glycation end-products (RAGE) gene and circulating RAGE levels as a susceptibility factor for 
non-alcoholic steatohepatitis (NASH)," PLoS One, vol. 13, no. 6, Article ID e0199294, 2018.

[69] H. Kan, S.-i. Yamagishi, A. Ojima et al., "Elevation of serum levels of advanced glycation end products in patients with non-B or non-C hepatocellular carcinoma," Journal of Clinical Laboratory Analysis, vol. 29, no. 6, pp. 480-484, 2015.

[70] F. Santilli, P. Blardi, C. Scapellato et al., "Decreased plasma endogenous soluble RAGE, and enhanced adipokine secretion, oxidative stress and platelet/coagulative activation identify non-alcoholic fatty liver disease among patients with familial combined hyperlipidemia and/or metabolic syndrome," Vascular Pharmacology, vol. 72, pp. 16-24, 2015.

[71] S.-C. Su, M.-J. Hsieh, Y.-E. Chou, W.-L. Fan, C.-B. Yeh, and S.-F. Yang, "Effects of RAGE gene polymorphisms on the risk and progression of hepatocellular carcinoma," Medicine, vol. 94, no. 34, p. e1396, 2015.

[72] E. D’Adamo, M. L. Marcovecchio, C. Giannini et al., "Improved oxidative stress and cardio-metabolic status in obese prepubertal children with liver steatosis treated with lifestyle combined with Vitamin E," Free Radical Research, vol. 47, pp. 146-153, 2013.

[73] K. H. J. Gaens, P. M. G. Niessen, S. S. Rensen et al., "Endogenous formation of $\mathrm{Ne}$-(carboxymethyl)lysine is increased in fatty livers and induces inflammatory markers in an in vitro model of hepatic steatosis," Journal of Hepatology, vol. 56, no. 3, pp. 647-655, 2012.

[74] E. D'Adamo, C. Giannini, V. Chiavaroli et al., "What is the significance of soluble and endogenous secretory receptor for advanced glycation end products in liver steatosis in obese prepubertal children?," Antioxidants and Redox Signaling, vol. 14, no. 6, pp. 1167-1172, 2011.

[75] Y. Kimura, H. Hyogo, S.-I. Yamagishi et al., "Atorvastatin decreases serum levels of advanced glycation endproducts (AGEs) in nonalcoholic steatohepatitis (NASH) patients with dyslipidemia: clinical usefulness of AGEs as a biomarker for the attenuation of NASH," Journal of Gastroenterology, vol. 45, no. 7, pp. 750-757, 2010.

[76] Y. Yilmaz, E. Ulukaya, O. O. Gul et al., "Decreased plasma levels of soluble receptor for advanced glycation endproducts (sRAGE) in patients with nonalcoholic fatty liver disease," Clinical Biochemistry, vol. 42, no. 9, pp. 802-807, 2009.

[77] H. Hyogo, S.-I. Yamagishi, K. Iwamoto et al., "Elevated levels of serum advanced glycation end products in patients with non-alcoholic steatohepatitis," Journal of Gastroenterology and Hepatology, vol. 22, no. 7, pp. 1112-1119, 2007.

[78] K. Asadipooya, "Letter to the editor: "advanced glycation end products and esRAGE are associated with bone turnover and incidence of hip fracture in older men"," The Journal of Clinical Endocrinology and Metabolism, vol. 104, no. 3, pp. 682-683, 2019.

[79] K. Asadipooya and E. M. Uy, "Advanced glycation endproduct, receptor for AGEs, diabetes and bone: review of literature," Journal of the Endocrine Society, vol. 3, no. 10, pp. 1799-1818, 2019.

[80] A. Eshraghian, "Current and emerging pharmacological therapy for non-alcoholic fatty liver disease," World Journal of Gastroenterology, vol. 23, no. 42, pp. 7495-7504, 2017.

[81] G. Brusotti, R. Montanari, D. Capelli et al., "Betulinic acid is a PPARgamma antagonist that improves glucose uptake, promotes osteogenesis and inhibits adipogenesis," Scientific Reports, vol. 7, p. 5777, 2017.

[82] K. Byun, Y. Yoo, M. Son et al., "Advanced glycation endproducts produced systemically and by macrophages: a common contributor to inflammation and degenerative diseases," Pharmacology and Therapeutics, vol. 177, pp. 44-55, 2017.

[83] P. Li, Z. Tang, L. Wang, and B. Feng, "Glucagon-like peptide-1 analogue liraglutide ameliorates atherogenesis via inhibiting advanced glycation end product-induced receptor for advanced glycosylation end product expression in apolipoprotein-E deficient mice," Molecular Medicine Reports, vol. 16, no. 3, pp. 3421-3426, 2017.

[84] D.-V. Nguyen, A. Linderholm, A. Haczku, and N. Kenyon, "Glucagon-like peptide 1: a potential anti-inflammatory pathway in obesity-related asthma," Pharmacology and Therapeutics, vol. 180, pp. 139-143, 2017.

[85] Z. Zhou, Y. Tang, X. Jin et al., "Metformin inhibits advanced glycation end products-induced inflammatory response in murine macrophages partly through AMPK activation and RAGE/NFkappaB pathway suppression," Journal of Diabetes Research, vol. 2016, Article ID 4847812, 10 pages, 2016.

[86] S. H. Ku, J. Hong, H.-H. Moon et al., "Deoxycholic acidmodified polyethylenimine based nanocarriers for RAGE siRNA therapy in acute myocardial infarction," Archives of Pharmacal Research, vol. 38, no. 7, pp. 1317-1324, 2015.

[87] M. B. Manigrasso, J. Pan, V. Rai et al., "Small molecule inhibition of ligand-stimulated RAGE-DIAPH1 signal transduction," Scientific Reports, vol. 6, p. 22450, 2016.

[88] T. Matsui, Y. Higashimoto, Y. Nishino, N. Nakamura, K. Fukami, and S.-i. Yamagishi, "RAGE-aptamer blocks the development and progression of experimental diabetic nephropathy," Diabetes, vol. 66, no. 6, pp. 1683-1695, 2017.

[89] D. Musumeci, G. N. Roviello, and D. Montesarchio, "An overview on HMGB1 inhibitors as potential therapeutic agents in HMGB1-related pathologies," Pharmacology and Therapeutics, vol. 141, no. 3, pp. 347-357, 2014.

[90] N. Mendez-Sanchez, V. C. Cruz-Ramon, O. L. Ramirez-Perez, J. P. Hwang, B. Barranco-Fragoso, and J. Cordova-Gallardo, "New aspects of lipotoxicity in nonalcoholic steatohepatitis," International Journal of Molecular Sciences, vol. 19, no. 7, p. 2034, 2018.

[91] D. B. Harmon, C. Wu, N. Dedousis et al., "Adipose tissue derived free fatty acids initiate myeloid cell accumulation in mouse liver in states of lipid oversupply," American Journal of Physiology Endocrinology and Metabolism, vol. 315, no. 5, 2018.

[92] Q. M. Anstee, H. L. Reeves, E. Kotsiliti, O. Govaere, and M. Heikenwalder, "From NASH to HCC: current concepts and future challenges," Nature Reviews Gastroenterology and Hepatology, vol. 16, no. 7, pp. 411-428, 2019.

[93] L. Xu, Y. R. Wang, P. C. Li, and B. Feng, "Advanced glycation end products increase lipids accumulation in macrophages through upregulation of receptor of advanced glycation end products: increasing uptake, esterification and decreasing efflux of cholesterol," Lipids in Health and Disease, vol. 15, no. 1, p. 161, 2016.

[94] W. Cai, J. Li, J. X. Xu et al., "Association of 2184AG polymorphism in the RAGE gene with diabetic nephropathy in Chinese patients with type 2 diabetes," Journal of Diabetes Research, vol. 2015, Article ID 310237, , 2015.

[95] P. Kang, C. Tian, and C. Jia, "Association of RAGE gene polymorphisms with type 2 diabetes mellitus, diabetic retinopathy and diabetic nephropathy," Gene, vol. 500, no. 1, pp. 1-9, 2012.

[96] J. Li, W. Cai, W. Zhang et al., "Polymorphism 2184A/G in the AGER gene is not associated with diabetic retinopathy in Han Chinese patients with type 2 diabetes," Journal of International Medical Research, vol. 44, no. 3, pp. 520-528, 2016. 


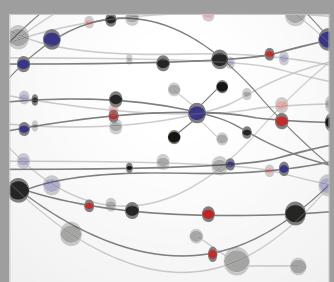

The Scientific World Journal
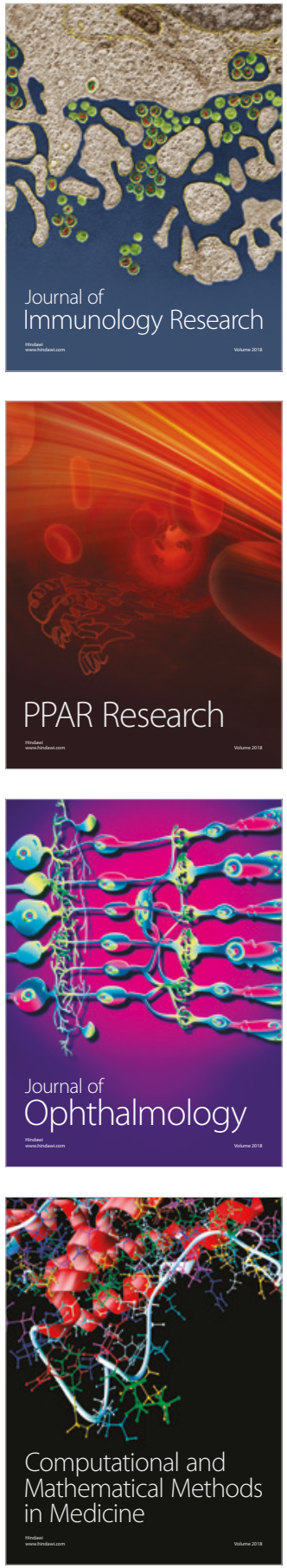

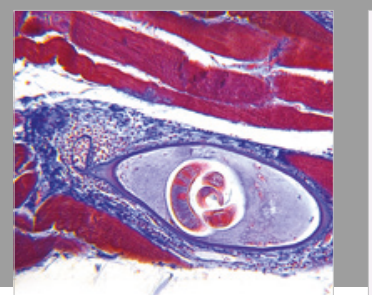

Gastroenterology Research and Practice

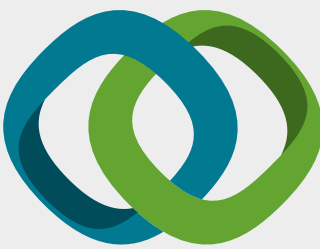

\section{Hindawi}

Submit your manuscripts at

www.hindawi.com
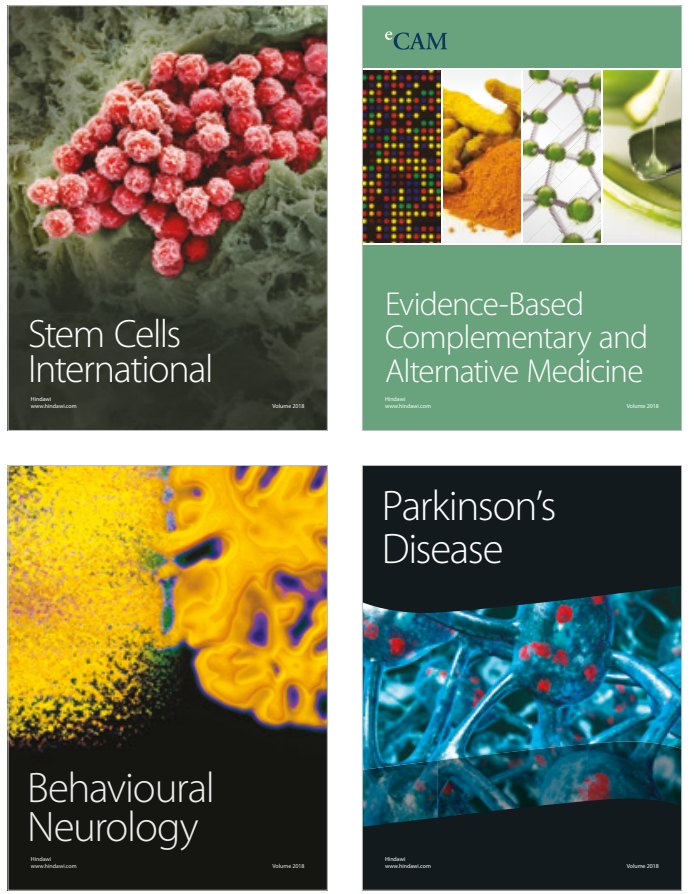

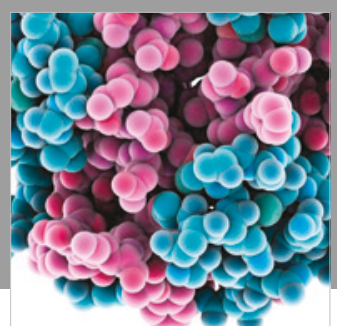

ournal of

Diabetes Research

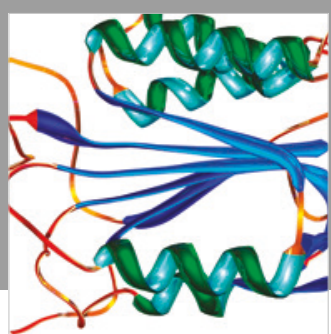

Disease Markers
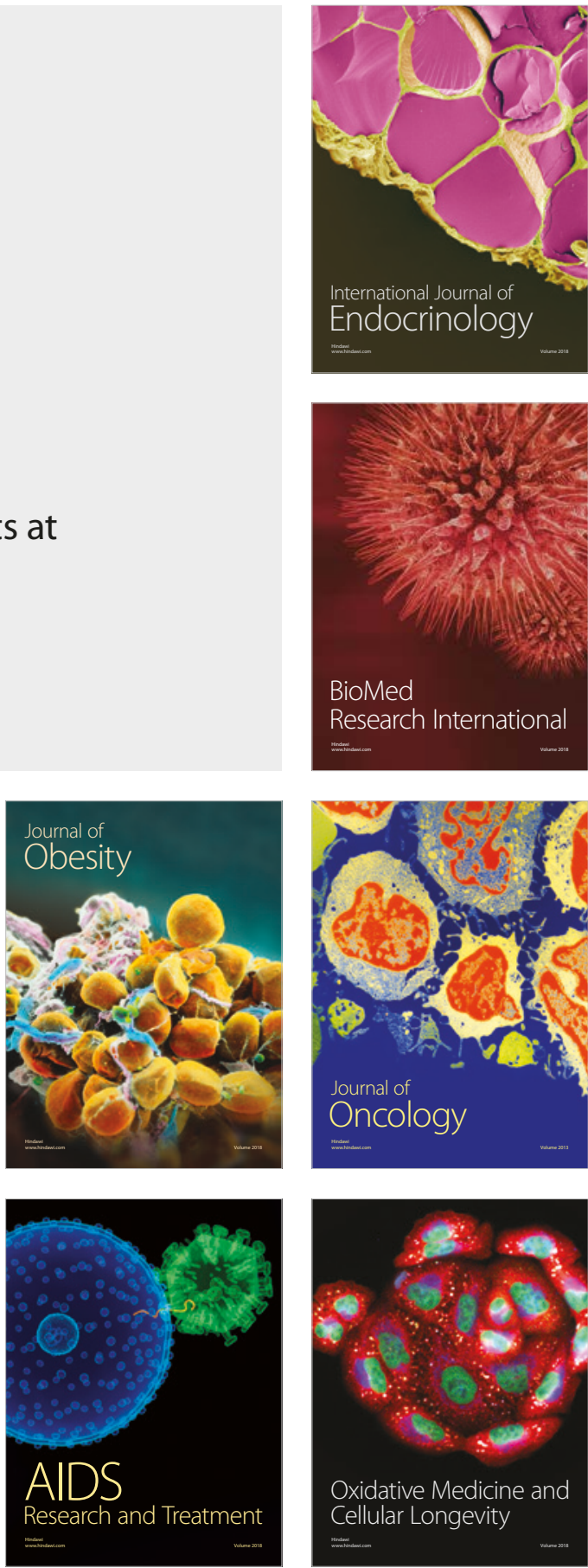